\title{
Transforming variant of Met receptor confers serum independence and anti-apoptotic property and could be involved in the mouse thymic lymphomagenesis
}

\author{
Cheol-Min Baek ${ }^{1}$, Soung-Hoo Jeon ${ }^{1, *}$, \\ Ja-June Jang ${ }^{3}$, Bok Soon Lee Le, $^{1,2}$ \\ and Jae-Ho Lee $e^{1,2,4}$ \\ ${ }^{1}$ Department of Biochemistry
${ }^{2}$ Chronic Inflammatory Disease Research Center
Ajou University Medical School
San 5 Woncheon-dong, Yeongtong-gu
Suwon 443-721, Korea
${ }^{3}$ Department of Pathology
Seoul National University College of Medicine
Seoul 110-799, Korea
${ }^{4}$ Corresponding author: Tel, 82-31-219-5053;
Fax, 82-31-219-5059; E-mail, jhlee64@ajou.ac.kr
${ }^{*}$ Present address: Department of Biotechnology, College of
Engineering, Yonsei University, Seoul 120-749, Korea \\ Accepted 30 May 2004
}

Abbreviations: EGF, epidermal growth factor; IL-6, interleukin 6; PKC, protein kinase C; PTP, protein tyrosine phosphatase; Stat, signal transducer and activator of transcription

\begin{abstract}
Met tyrosine kinase receptor, the receptor of hepatocyte growth factor/scatter factor (HGF/SF), is present in mouse tissues as two major isoforms differing by a 47-aminoacid segment in the juxtamembrane domain via alternative splicing of exon 14. We found that the smaller isoform of Met (Sm-Met) was highly transformable in both in vitro and in vivo tumorigenesis assays. In this report, close examination of the transforming activity of the Sm-Met showed that the expression of Sm-Met conferred the cells serum independence and antiapoptotic property when treated with doxorubicin. These properties of Sm-Met seemed to be originated from its far longer maintenance of tyrosine kinase activity after the binding of HGF/SF. Interestingly, the longer maintenance of activated status was accompanied with more increase of tyrosine phosphorylation of Stat3 protein. Moreover, we have tried to find (an) animal tumorigenesis model(s) showing the increase in the expres-
\end{abstract}

sion of this transforming variant of Met. In $\gamma$ ray-induced mouse thymic lymphoma model, the expression of the mRNAs for Sm-Met was significantly increased as well as those of wild type Met and HGF/SF, suggesting a possible role of the Sm-Met in tumorigenesis in vivo.

Keywords: alternative splicing; anti-apoptosis; hepatocyte growth factor; lymphoma; Met; proliferation; Stat3

\section{Introduction}

The Met tyrosine kinase is a high-affinity receptor for hepatocyte growth factor/scatter factor (HGF/SF) (Bottaro et al., 1991; Naldini et al., 1991). Both Met and HGF/SF are expressed in numerous tissues, although their expression is confined predominantly to cells of epithelial and mesenchymal origin, respectively (Stoker et al., 1987). Signaling via this receptorligand pair has been shown to affect a wide range of biological activities, including angiogenesis (Bussolino et al., 1992), cellular motility (Stoker et al., 1987), growth (Nakamura et al., 1986), and morphogenic differentiation (Montesano et al., 1991; Tsarfaty et al., 1992).

In addition to mediating a variety of normal cellular processes, Met-HGF/SF signaling has also been implicated in the generation and spread of tumors (reviewed in Jeffers et al., 1996a). In this regard, several oncogenic forms of Met have been discovered. The tpr-met oncogene is one such form of the Met receptor, and it represents the fusion of two distinct genetic loci: tpr which encodes a proteinprotein dimerization motif, and met which encodes an intracellular portion of the Met receptor, including its kinase domain (Cooper et al., 1984; Park et al., 1986). The resultant chimeric protein homodimerizes and transphosphorylates each other, thereby generating molecules possessing constitutive kinase activity and transforming ability.

A second oncogenic form of Met is the native unaltered receptor itself following continual HGF/SF autocrine stimulation (Jeffers et al., 1996b). In addition to mediating transformation in model systems, there is evidence that autocrine Met-HGF/SF signaling may play a role in human cancer (reviewed in Jeffers et al., 1996a). Finally, Met can be oncogenically 
activated via a number of specific point mutations. Mutations originally identified in human papillary renal carcinomas (Schmidt et al., 1997) were found to generate Met molecules possessing constitutive kinase activity and transforming ability (Jeffers et al., 1997). In addition to papillary renal carcinoma, mutation of Met was reported in one case of ovarian cancer (Tanyi et al., 1999), in some cases of early-onset hepatocellular carcinoma (Park et al., 1999) and in gastric carcinoma by the authors (Lee et al., 2000). Whereas all the mutations reported in hereditary papillary renal cancer and hepatocellular carcinoma are missense mutations of tyrosine kinase domain of Met, the missense mutation we found from gastric carcinoma was located in juxtamembrane domain and showed transforming activity when overexpressed in NIH3T3 mouse fibroblasts (Lee et al., 2000). Along with some reports showing the presence of protein kinase C (PKC) regulatory site (Gandino et al., 1994) and phosphotyrosine phosphatase binding site (VillaMoruzzi et al., 1998) in juxtamembrane domain, the location of the mutation in juxtamembrane domain strongly suggested possible significance of juxtamembrane domain in Met-mediated tumorigenesis (Figure 1).

Lee et al. reported an alternative splicing form of Met (Sm-Met), which doesn't have part of juxtamembrane domain due to the skipping of exon 14 of met gene (Lee and Yamada, 1994), in which part all the known regulatory sites of Met tyrosine kinase resides (Figure 1). Although initial report for the presence of the Sm-Met came early, there has been

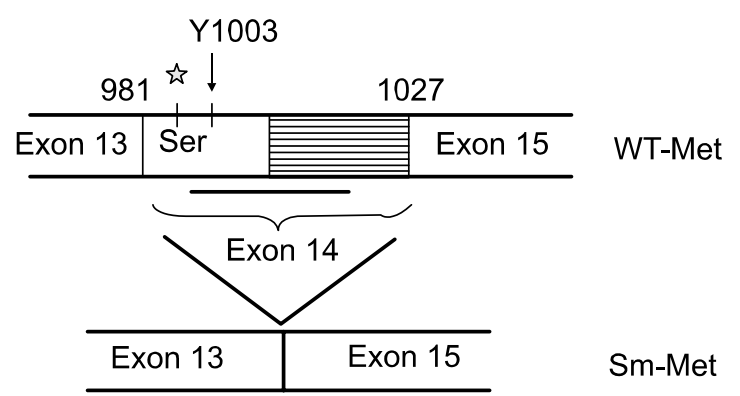

Exon 14 has four putative regulatory sites

$$
\begin{aligned}
& \text { ఓ: : Tyrosine kinase activity down-regulation by PKC } \\
& \text { (J Biol Chem 1994 Jan 21;269(3):1815-20) } \\
& \text { Е: Binding site of PTP-S (Biochem J 1998 Nov 15;336(Pt 1):235-9) } \\
& \text { —: PEST sequence } \\
& \downarrow \text { : c-Cbl binding site (Mol Cell } 2001 \text { Nov;8(5):995-1004) }
\end{aligned}
$$

Figure 1. Putative regulatory sites localized in juxtamembrane region encoded by exon 14. This is a schematic representation showing the putative regulatory sites of Met. Exon 14 of Met contains 4 different putative regulatory sites including Ser985 phosphorylation site by PKC, PTP-S binding site, PEST sequence and $\mathrm{c}-\mathrm{Cbl}$ binding site. no reports on the physiological or pathological meaning of this isoform yet to the best of our knowledge. We surmised that if a point mutation in juxtamembrane domain can induce tumorigenesis possibly by the derangement of proper regulation of tyrosine kinase activity of Met, deletion of entire exon which harbors the entire regulatory activity should induce drastic changes in Met tyrosine kinase activity.

Indeed, we could observe the strong transforming activity of Sm-Met and its biochemical difference from wild type, suggesting a possible new mode of Metrelated tumorigenesis (submitted to elsewhere). In this report, the authors have found that the expression of Sm-Met conferred the cells serum independence and anti-apoptotic property. These properties of Sm-Met seemed to be originated from its longer maintenance of tyrosine kinase activity followed by more increase of tyrosine phosphorylation of Stat3 protein. Moreover, we showed that the expression of the mRNAs for Sm-Met was significantly increased in $\gamma$-rayinduced mouse thymic lymphoma model, suggesting a possible involvement of the Sm-Met in tumorigenesis in vivo.

\section{Materials and Methods}

\section{Cell lines}

NIH3T3 cells (CRL1658) were obtained from the American Type Culture Collection (ATCC) and cultured in DMEM (Gibco-BRL, Rockville, MD) supplemented with $10 \%$ (V/V) calf serum (CS) (Gibco-BRL). These cells produce 1-2 $\mathrm{ng} / \mathrm{ml}$ of $\mathrm{HGF} / \mathrm{SF}$, of which the level is comparable to the concentration of HGF/ SF in normal serum (Han et al., 1999).

\section{Constructs}

The wild-type Met expression vector contains the murine Met cDNA of T1010l polymorphism in PMB1 as described previously (Lee et al., 2000). The Sm-Met expression vector was constructed by using the original clone (Lee and Yamada, 1994) into the same expression vector as wild type. To construct P1009S mutant of Met, the QuickChange site-directed mutagenesis kit (Stratagene, La Jolla, CA) was used according to a manufacturer's instructions with PMB11 as the template. Mutations were verified by sequencing both strands of DNA in the region of interest.

\section{Reagents, growth factor and antibodies}

All reagents used were from Sigma (Saint Louis, MO). Rabbit anti-murine Met antibody (SP260) was purchased from Santa Cruz (Santa Cruz, CA). Mouse anti-phosphotyrosine antibody (4G10) was purchased 
from Upstate Biotechnologies (Lake Placid, NY). Recombinant human $\mathrm{HGF} / \mathrm{SF}$ and neutralizing antibody for HGF/SF were purchased from R\&D systems (Minneapolis, MN).

\section{Transfection and screening}

Transfections and screening were performed as described previously (Lee et al., 2000) by using lipofectamine (Gibco-BRL). The transfected cells were cultured in DMEM $110 \%$ CS supplemented with $800 \mu \mathrm{g} / \mathrm{ml}$ G-418 (Gibco-BRL) for two weeks. These cells were used as pools of cells consisting of at least 100 colonies for further experiments.

\section{Immunoprecipitation}

Monolayers of stably transfected cells were washed twice with ice-cold PBS, lysed in ice-cold RIPA buffer consisting of $10 \mathrm{mM}$ sodium phosphate $(\mathrm{pH} 7.2), 150$ $\mathrm{mM} \mathrm{NaCl}, 1 \%$ (V/V) Nonidet P-40, 0.1\% (W/V) SDS supplemented with $10 \mathrm{mM}$ sodium fluoride, $5 \mathrm{mM}$ sodium orthovanadate, and complete protease inhibitor cocktail (Boehringer Mannheim, Germany), and centrifuged $\left(15 \mathrm{~min}, 4^{\circ} \mathrm{C}, 14,000 \mathrm{~g}\right)$. After quantitation by using BCA protein assay reagent (Pierce, Rockford, IL), $400 \mu \mathrm{g}$ of each lysate was precleared with protein A-sepharose and then incubated with anti-Met antibody (SP260) and protein A-Sepharose for overnight at $4^{\circ} \mathrm{C}$ with rotation. The samples were then washed three times with ice-cold RIPA buffer. SDS gel-loading buffer (containing reducing agent) was added to each sample. After boiling $(5 \mathrm{~min})$ and centrifuging (5 $\mathrm{min}, 14,000 \mathrm{~g}$ ), the resulting supernatants were resolved by SDS/PAGE and examined by Western blotting.

\section{Western blotting}

Western blotting was performed essentially as described (Jeffers et al., 1996b) under reducing conditions using the following primary antibodies: rabbit anti-Met polyclonal antibody (SP260; Santa Cruz), anti-phosphotyrosine monoclonal antibody (clone 4G10; Upstate Biotechnologies).

\section{Thymic lymphoma model}

Thymic lymphoma was induced in female C57BL/6J mice with slight modifications of other groups' report (Brathwaite et al., 1992). Briefly, beginning at 5 weeks of age, mice were irradiated using whole body $\gamma$-irradiation protocol (5 times, 1.9 Gy/exposure, once a week). One age-matched control animal and groups of treated animals were sacrificed at 6 months and 10 months after the irradiation. The thymus tissue was removed, weighed, and verified for the presence of lymphoma by a pathologist. Tissues obtained at 10 months after irradiation were used in this experiment.

\section{RNA extraction and reverse-transcription polymerase chain reaction (RT-PCR)}

Total cellular RNA isolated from indicated cells, normal thymus or thymic lymphoma tissues with Trizol reagent (Gibco-BRL) were applied for cDNA synthesis using Moloney murine leukemia virus reverse transcriptase (Bioneer, South Korea) and oligo-dT as primers. Both WT- and Sm-Met cDNA fragments were amplified using the following primers: forward; 5'-CCG AAGTGTAAGTCCAATTACAGAGATGG-3', reverse; 5'CCATCTCTGTAATTGGACTTACACTTCGg-3'. Primers used for the amplification of HGF/SF were: forward; 5'-CCATGAATTTGACCTCTATG-3, reverse; 5'-ACTGAGGAATGTCACAGAC-3'. As for actin, forward; 5'-CAGGTCCAGACGAGGATGGCAT-3', reverse; 5'CGACATGGAGAAAATCTGCACC-3'. The reaction mixture $(50 \mu \mathrm{l})$ contained $1.5 \mathrm{mM} \mathrm{MgCl}_{2}, 25 \mu \mathrm{M}$ of each dNTP, and 1 pmole of each oligonucleotide primer in buffer and incubated under the program of $94^{\circ} \mathrm{C} \times 5 \mathrm{~min}$; then 30 cycles of $94^{\circ} \mathrm{C} \times 30 \mathrm{~s}, 53^{\circ} \mathrm{C} \times 30$ s, $72^{\circ} \mathrm{C} \times 45 \mathrm{~s}$; ending with $72^{\circ} \mathrm{C} \times 10 \mathrm{~min}$ in the thermal cycler (Perkin Elmer, Boston, MA). Met expression was amplified for 30 cycles. The PCR products were then analyzed by $1 \%$ agarose gel electrophoresis.

\section{Cell proliferation}

Pools of NIH3T3 cells expressing comparable amounts of the two exogenous proteins (WT-Met and $\mathrm{Sm}$-Met) were tested in a proliferation assay. Cells were plated at low density and then starved in low serum condition $(0.2 \%$ CS $)$. Cell proliferation was evaluated at indicated times by cell counting or by using Cell Proliferation Kit I (Roche Molecular Biochemicals, Indianapolis, IN) which was based on the MTT assay according to the manufacturer's instruction. In order to evaluate the effect of endogenous HGF/SF secreted by NIH3T3 cells, anti-HGF/SF neutralizing antibody (R\&D) was used when indicated.

\section{Anti-apoptotic activity}

Cells were cultured in 96-well plates at a density of 2,000 cells/well. Twenty-four $h$ after seeding, cells were washed with serum-free media and then media containing different doses of HGF/SF (with $0.2 \%$ FBS) was added to each well. Twenty-four $h$ later, doxorubicin was added to each well at the indicated concentrations. Cell Proliferation Kit I (Roche Molecular Biochemicals) was used to measure the number of survived cells. In order to correct the effect of mitogenic activity of HGF/SF on this assay, absorb- 
ance at each well was divided by the cell proliferating ratio (absorbance at HGF/SF-treated well without doxorubicin treatment/absorbance at HGF/SF-nontreated well without doxorubicin treatment). The antiapoptotic activity of HGF/SF was calculated as the corrected absorbance value/absorbance value at the well without HGF/SF treatment.

\section{Results}

\section{Sm-Met decreases serum-dependence of NIH3T3 cells}

We found that the Sm-Met could transform NIH3T3 cells strongly when assessed by foci-forming assay and agarose-colony generation assay. Furthermore, the cells over-expressing this isoform strongly induced tumorigenesis in nude mice (result not shown). To dissect the transforming activity of Sm-Met, change in the serum-dependence was assessed first. As shown in Figure 2, pools of NIH3T3 cells expressing comparable amounts of the two exogenous proteins (WT-Met and Sm-Met; data not shown) were tested in a proliferation assay. Cells expressing Sm-Met grew significantly faster than WT-Met expressing cells at low serum concentration (Figure 2A). On the contrary, there was no significant difference in proliferation between two in the presence of $10 \% \mathrm{CS}$ (data not shown). Since NIH3T3 cells are known to secrete HGF/SF, the difference observed might come from either their different responsiveness to secreted HGF/SF or from their different responsiveness to serum. In order to address that, we treated cells with Wt-Met with HGF/SF while cells with Sm-Met were treated with neutralizing anti-HGF/SF antibody. As shown in Figure 2B, treatment with exogenous HGF/ SF made the cells with WT-Met proliferate as fast as the cells with Sm-Met. Also, treatment with anti-HGF/ SF decreased the proliferation of the cells with SmMet to the level observed in cells with WT-Met. It is highly probable that the difference in proliferation between two cells come from their different responsiveness to endogenous HGF/SF.

\section{Sm-Met has anti-apoptotic property}

HGF/SF has been reported to provide anti-apoptotic activity to the cells. We next move to anti-apoptotic activity to explain the transforming activity of Sm-Met. A chemotherapeutic agent, doxorubicin (adriamycin) was treated to WT- or Sm-Met transfected cells with or without pre-treatment with HGF/SF. As shown in Figure 3 , doxorubicin exerted cytotoxic effect to the cells with WT-Met dose-dependently, and the cytotoxicity was effectively blocked by the administration of HGF/SF. Interestingly, cells with Sm-Met revealed re
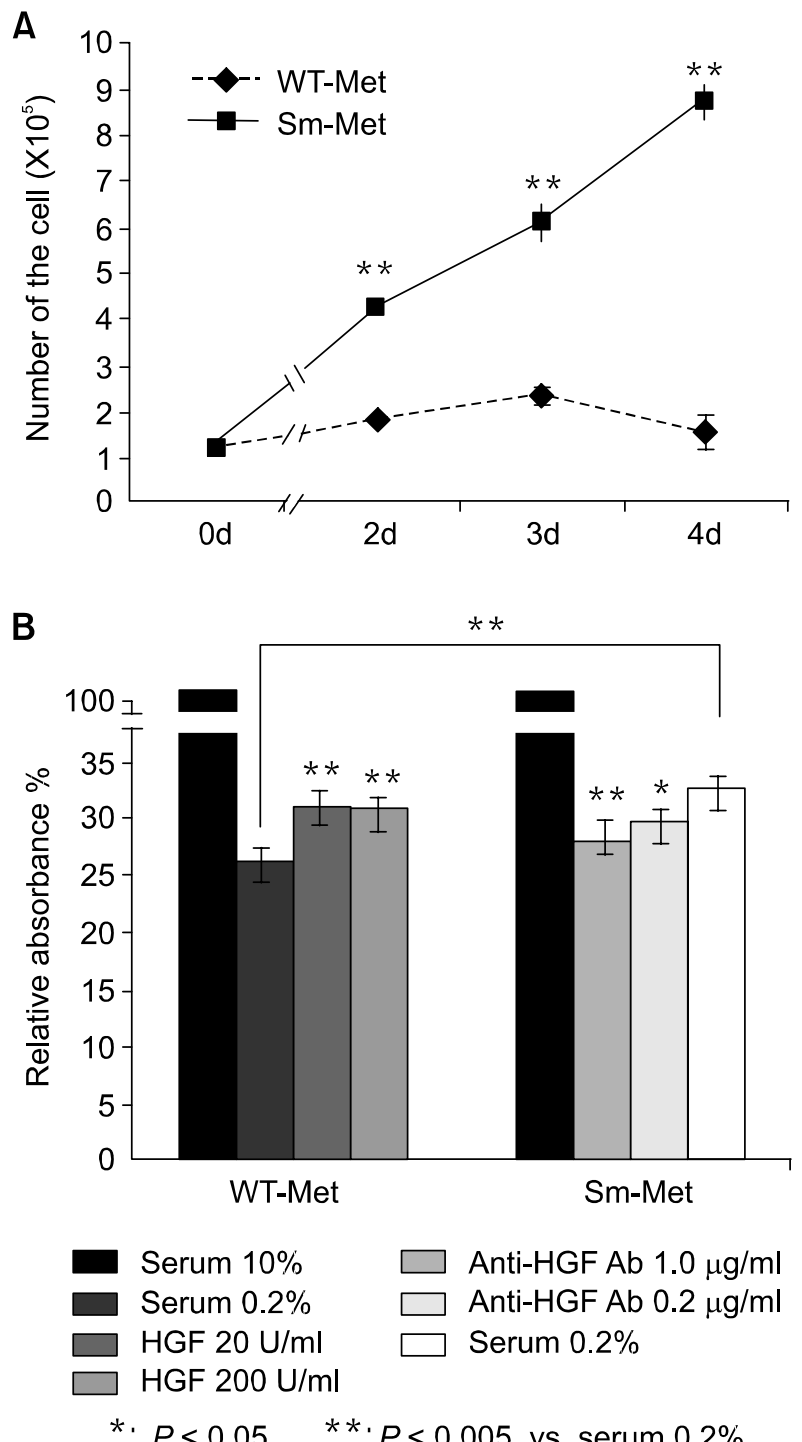

Figure 2. Serum-independent growth by Sm-Met expression. Pools of clones expressing WT- or Sm-Met proteins were tested in proliferation assay. (A) WT- or Sm-Met transfected cells $\left(1.2 \times 10^{5}\right.$ cells each) were seeded in $60 \mathrm{~mm}$ dish. Cells were cultured in Dulbecco's modified Eagle's medium (DMEM) with 10\% calf serum (CS). After $24 \mathrm{~h}$, cells were washed twice with serum free DMEM and supplemented with $0.2 \%$ CS and the live cells were counted at the indicated days. Reported numbers are means of quadruplicates (+/- standard deviation). (B) To block the HGF/SF activity, indicated amount of anti-HGF/SF neutralizing antibody was added to DMEM containing $0.2 \% \quad C S$. To address the effect of full activation of $\mathrm{HGF} / \mathrm{SF}$, indicated amount of activated HGF/SF was added to the media. DMEM with $0.2 \%$ CS containing the indicated concentration of HGF/SF or anti- HGF/SF neutralizing antibody was applied to the respective cells. MTT assay was performed after 2 days. ${ }^{*} P<0.05$, ${ }^{* *} P<0.005$ (both vs. $0.2 \%$ serum condition by Student's $t$-test). 


\begin{tabular}{|c|c|c|c|c|}
\hline Cell seeding & $\begin{array}{l}\text { Starvation or } \\
\text { HGF treatment }\end{array}$ & $\begin{array}{l}\text { Adriamycin } \\
\text { treatment }\end{array}$ & MTT assay & $A_{570 \mathrm{~nm}}$ \\
\hline & $\uparrow$ & $\uparrow$ & Washing \& $\uparrow$ & $\uparrow$ \\
\hline & $24 \mathrm{~h}$ & $24 \mathrm{~h} \quad 2 \mathrm{~h}$ & starvation $24 \mathrm{~h}$ & $24 \mathrm{~h}$ \\
\hline
\end{tabular}

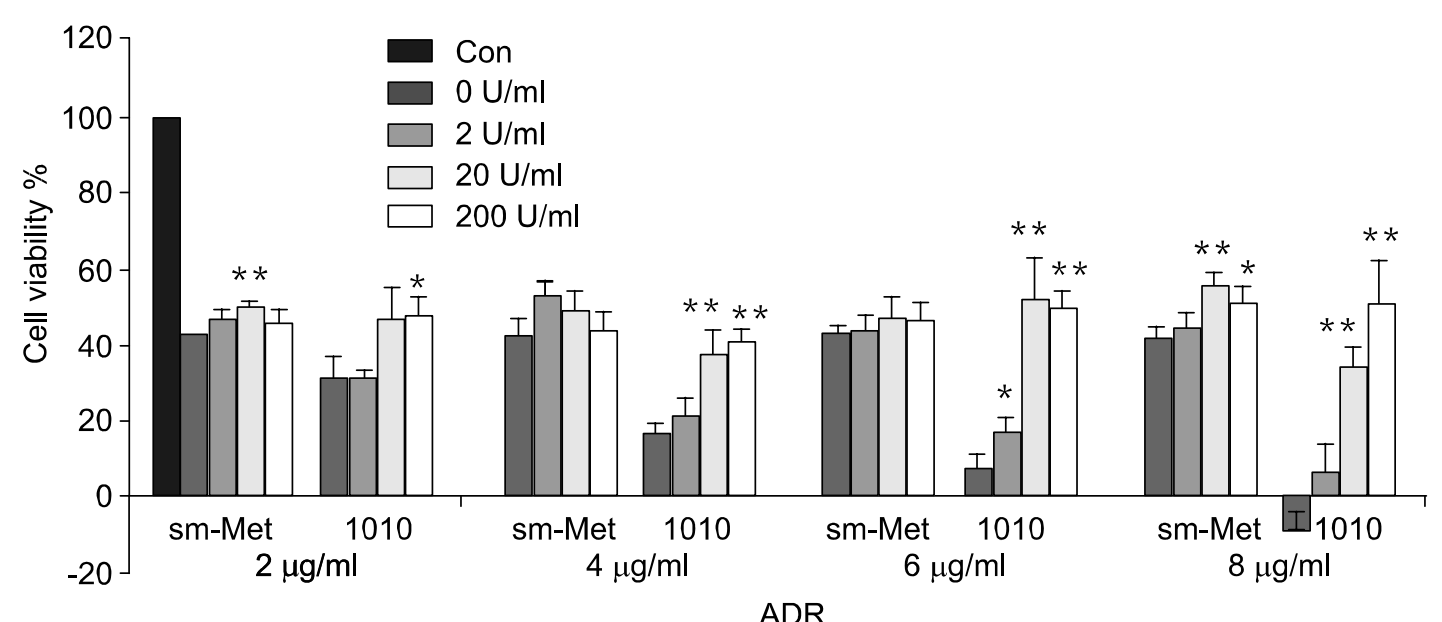

Figure 3. Anti-apoptotic activity of Sm-Met. Twenty-four $h$ after seeding $2.0 \times 10^{3}$ cells per well, cells were cultured in DMEM containing $0.2 \%$ CS with indicated concentrations of HGF/SF followed by treatment with adriamycin at indicated concentrations for $2 \mathrm{~h}$. Cells were washed twice with serum-free DMEM and supplemented with DMEM containing $0.2 \% \mathrm{CS}$. After $24 \mathrm{~h}$, cell viability was assessed by using MTT assay $\left({ }^{*} P<0.05,{ }^{* *} P<0.005\right.$ vs. $0 \mathrm{U} / \mathrm{ml}$ condition by Student's $t$-test).

sistance to doxorubicin even without exogenous HGF/ SF. Intriguingly, high level of doxorubicin could not increase the death of the Sm-Met transfected cells.

\section{Biochemical difference between WT- and Sm-Met}

To find out the basic mechanism underlying the difference in biological activity, more than 100 clones of WT-, P1009S mutant-, or Sm-Met transfected NIH3T3 colonies were pooled and analyzed biochemically. To address whether there is a quantitative difference in Met signaling, the amount of tyrosine phosphorylation of Met protein was evaluated because it has been well-recognized that the activity of the tyrosine kinase of Met is well-correlated with the amount of phosphotyrosine on Met protein (Naldini et al., 1991). We expected the difference in the amount of tyrosine phosphorylation of Met since all known regulatory mechanism of Met reside in juxtamembrane domain encoded by exon-14. In the absence of exogenous HGF/SF, the amount of phosphotyrosine on Met protein was not significantly different between WT- and Sm-Met (Figure 4). However, $24 \mathrm{~h}$ after the addition of exogenous HGF/SF, the phosphotyrosine content of WT-Met was indeed far less than those of both P1009S mutant and Sm-Met (Figure 4, upper panel). When the same blot was stripped and Met protein was analyzed, the amount of the protein was not different enough to explain the difference in

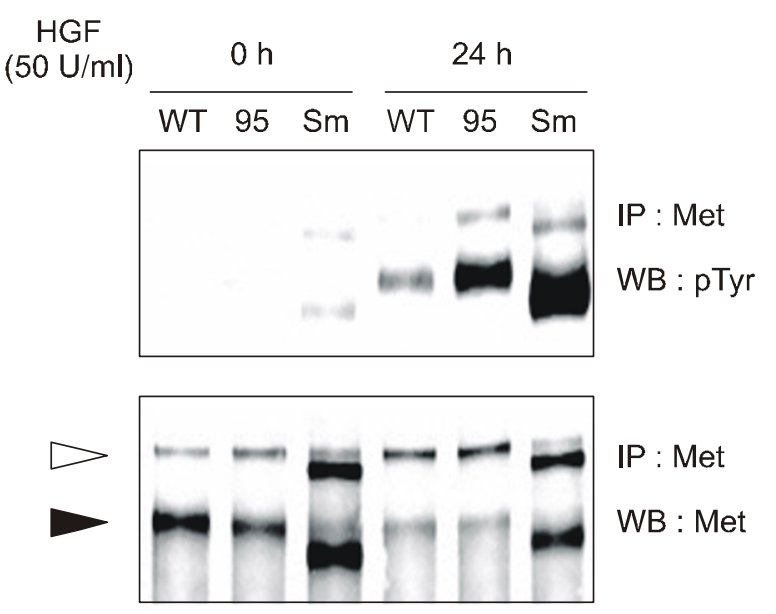

Figure 4. Difference in the amount of tyrosine pnospnorylation upon HGF/SF treatment between WT- and Sm-Met. Samples labeled WT, 95 and $\mathrm{Sm}$ are from cells stably transfected with vectors expressing WT-, P1009S mutant and Sm-Met, respectively. Cells were cultured in DMEM/10\%CS to the confluency of $40-50 \%$. HGF/SF (50 scatter $\mathrm{U} / \mathrm{ml}$ ) was added to each dish and the cells were harvested $24 \mathrm{~h}$ later. Four hundred micrograms of cell lysate/sample were immunoprecipitated with anti-Met antibody (SP260), resolved on an $8 \%$ gel, and analyzed by Western analysis using anti-phosphotyrosine antibody (upper panel). The filters were stripped and reprobed with anti-Met antibody (lower panel). Open and closed triangle denote the single chain precursor form of Met, p170Met and the b-chain of the mature Met heterodimer, p140Met, respectively. 
tyrosine phosphorylation (Figure 4 lower panel)

\section{The amount of the tyrosine phosphorylation of STAT3 is different between WT- and small forms}

In order to find out the differences between WT- and Sm-Met in terms of intracellular signal transduction, WT- and Sm-Met transfected cells were treated with HGF/SF, and the lysates were analyzed by Western blotting. Since growth factor-induced cell proliferation and anti-apoptotic activities were known to be attributed to ERK, AKT or STAT3 proteins in various sys-

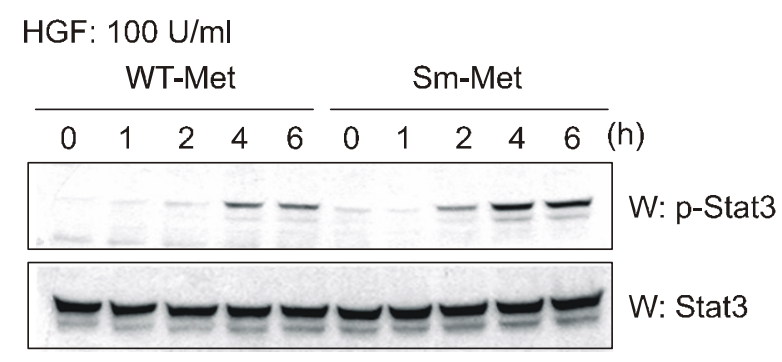

Figure 5. Difference in the degree of tyrosine phosphorylation of Stat3 between WT- and Sm-Met transfected cells. Cells were grown in DMEM/10\%CS to the $40-50 \%$ confluence. HGF/SF (100 scatter U/ $\mathrm{ml}$ ) was added to each dish at various time points before the harvest as indicated. Cell lysates were resolved on an $8 \%$ gel, and analyzed by Western blothing using anti-phosphoStat3 antibody (upper panel). The filters were stripped and reprobed with anti-Stat3 antibody (lower panel).

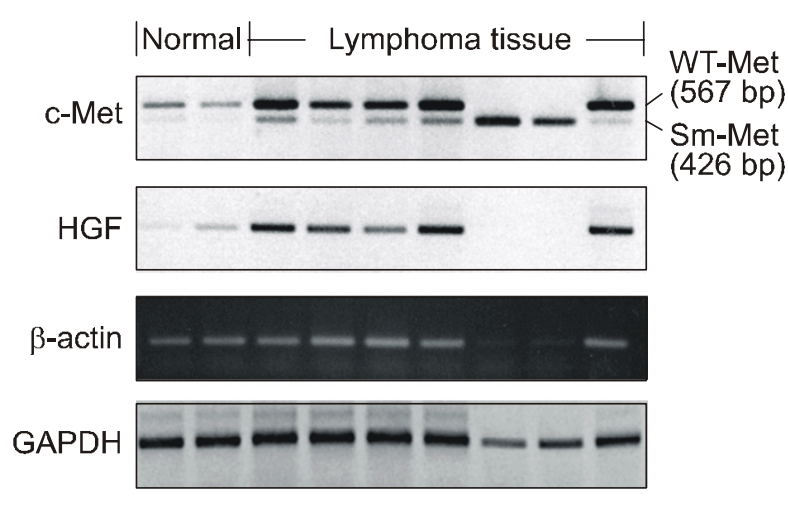

Figure 6. Change in the expressions of WT- and Sm-Met mRNA during $\gamma$-irradiation-induced thymic lymphomagenesis. Thymic lymphoma was induced by $\gamma$-irradiation as described in Materials and Methods. Total RNA was extracted by using RNAzoL from the lymphoma tissues and control normal mouse thymus without irradiation. RT-PCR analysis by using common primers for WT- and Sm-met was performed, and the resultant PCR product was analyzed by agarose gel electrophoresis. Upper band from upper panel denotes the PCR product for WT-Met mRNA and the lower band denotes that from $\mathrm{Sm}$-Met mRNA. Expression of HGF/SF mRNA was also assessed. tems, activation of those pathways were assessed by using phospho-specific antibodies. Whereas phosphorylation levels of ERK and AKT were not significantly different between two samples (data not shown), that of STAT3 seemed to be significantly different between them (Figure 5). Interestingly, the phosphorylated form of STAT3 increased quite obviously $2 \mathrm{~h}$ after the treatment of ligand.

\section{Sm-Met mRNA was increased in mouse thymic lymphoma tissues}

Since the Sm-Met induced cellular transformation in $\mathrm{NIH} 3 \mathrm{~T} 3$ cells and showed proliferative and antiapoptotic activities in cells, it would be fair to expect the contribution of this alternative splicing form in tumorigenesis in vivo. We have checked a few murine carcinogenesis models including $\gamma$-irradiation-induced mouse thymic lymphoma model for that purpose by RT-PCR analysis. Indeed, as shown in Figure 6, the amount of Sm-Met mRNA significantly increased in thymic lymphoma tissues compared to normal thymus. In addition, the expression level of both HGF/ SF and WT-Met also increased significantly in tumor tissues, suggesting that the overall increase of MetHGF/SF signaling may be highly related with thymic lymphomagenesis. The increased expression of $\mathrm{Sm}$ Met alternative splicing form might contribute to the overall increase of Met-HGF/SF signaling.

\section{Discussion}

Sm-Met has been first reported from mouse as an alternative splicing form of Met having deletion of 47 aminoacids in the juxtamembrane domain (Lee and Yamada, 1994). The fact that the differential splicing and its resultant protein occurs in a variety of mouse tissues, suggesting biological significance of this isoform. We have tried to find out the Sm-Met from rat tissues and human tissues by RT-PCR. While the $\mathrm{Sm}-$ Met was present in various tissues from rat, it was not observable from any human normal tissues tested (unpublished observation by J.H. Lee). In addition to the possible difference between murine and human in terms of cellular factors related with alternative splicing, because a number of features in the pre-mRNA can contribute to alternative splice site selection, including intron size, the relative strength of $5^{\prime}$ splice sites, the pyrimidine content of the 3 ' splice sites, the location of branch points, the presence of multiple alternative branch points, and specific exon sequences (reviewed in Lopez, 1998), it is hard to speculate the probable reason of the difference between species. However, recently, some researchers claimed to find Sm-Met from human lung cancer 
samples (personal communication), suggesting possible involvement of this alternative splicing form in human tumorigenesis.

The role of juxtamembrane region of Met in cell transformation is relatively unknown. Regarding this, there was a report that by adding juxtamembrane domain to TPR-Met fusion protein, they could abolish the transforming activity of TPR-MET (Vigna et al., 1999 ), suggesting this region is doing some suppressive role on the transforming activity of activated Met. Actually, the part of juxtamembrane domain deleted in Sm-Met was believed to act as regulatory sites of the enzymatic activity of Met. Specifically, two sites have been described as regulatory sites. Ser985 (Ser1003 according to the numbering system based on Gene Bank \#J02958) was described as a substrate of PKC, and its phosphorylation by PKC results in the down-regulation of tyrosine kinase activity of Met (Gandino et al., 1994). In addition, there was a report that a specific protein tyrosine phosphatase, PTP-S, binds to this region and acts as a down- regulation mechanism after the activation of receptor by the binding of ligand (Villa-Moruzzi et al., 1998). The remarkable difference in terms of tyrosine phosphorylation level between two forms after adding HGF/SF strongly suggests the difference in down-regulation mechanism.

Stat3 is one of seven known STAT (Signal Transducers and Activators of Transcription) family members, and the initial finding that Stat3 was constitutively activated in $\mathrm{V}-\mathrm{Src}$ transformation ( $\mathrm{Yu}$ et al., 1995; Cao et al., 1996) suggested that aberrant STATs may have key roles in oncogenesis. Constitutively activated Stat 3 mutant alone is sufficient to induce transformation in nude mice (Bromberg et al., 1999). In addition to V-Src, other tyrosine kinases such as v-Fps, v-Eyk, and Lck activated Stat3 in the context of oncogenesis (reviewed in Bowman et al., 2000). Moreover, Stat3 activation has been reported to be associated with transformation by tumor viruses such as HTLV-1, polyoma virus middle $T$ antigen, and EBV (reviewed in Turkson et al., 2000). Several evidences suggest the central role of Stat3 in human malignancies. Constitutive activation of Stat3 has been detected in breast carcinomas, head and neck squamous cell carcinomas, etc (reviewed in Turkson et al., 2000). Since there has not been identification of a naturally-occurring dominant-active mutation in stat3 gene, it is reasonable to hypothesize that aberrant upstream signaling events are the major driving force for the induction of dysregulated Stat3 signaling that is observed in oncogenesis. One of those would be aberrant signaling from certain growth factor(s)/ receptor(s).

As for the HGF/SF-related tumorigenesis, Stat3 has been reported to provide an essential function for tumorigenic growth by activating mutant of Met (Zhang et al., 2002) found in human hereditary renal papillary cancer. Also there was a report demonstrating a role of Stat3 in transducing survival signals downstream of tyrosine kinases such as Src, EGF-R, and Met, as well as cytokines such as IL-6, in human nonsmall cell lung cancers (Song et al., 2003). Moreover, in papillary thyroid tumors, expression of the HGF/SF, Met, and Stat3 reached 100\% (Trovato et al., 2003).

HGF/SF has been known to confer proliferating activity and anti-apoptotic activity. As for the mediating signal transducers, ERK pathway was reported to be related with both proliferating and anti-apoptotic activity. PI3 kinase and Akt also were known to mediate anti-apoptotic activity in some cells (reviewed in Gohda, 2002). In addition, Stat1, Stat3, and Stat5, activated in different tissues by means of a series of ligands also play important roles in controlling cellcycle progression and apoptosis and thus contributes to oncogenesis (reviewed in Calo et al., 2003), which corroborates well with our data.

We have tried to see whether the expression of $\mathrm{Sm}$-Met changes during murine carcinogenesis process. Among the different models tested, increase of Sm-Met as well as WT-Met and HGF/SF was observed in $\gamma$-ray-induced thymic lymphomagenesis model. Lymphoma has been reported to be related with Met-HGF/SF signaling. Serum HGF/SF level was significantly elevated both in Hodgkin's and nonHodgkin's lymphoma cases (Giles et al., 2004). In addition, high level of serum HGF/SF was correlated with multiple extra-nodal involvement and progression of the lymphoma (Hsiao et al., 2003), suggesting the level of HGF/SF is related with not only tumorigenesis but also progression in this tumor. Interestingly, there was a report that HGF/SF protects Burkitt lymphoma cell lines from apoptotic death induced by DNA damaging agents such as doxorubicin and etoposide (Skibinski et al., 2001), corroborating our data that Sm-Met significantly protected the cells from the apoptotic activity of doxorubicin, and also were associated with the thymic lymphomagenesis.

Many different tumors have been reported to be related with aberrant activation of Met-HGF/SF signaling via amplification of the ligand and/or receptor, aberrant expression of the ligand or receptor resulting in the formation of autocrine loop, or activating mutation of the receptor. We report the proliferating and anti-apoptotic activity of Sm-Met and its biochemical difference from wild type, which probably result in the difference in Stat3 signaling. In addition, the increase of the mRNA for Sm-Met in murine lymphomagenesis model suggests a possible new mode of Met-related tumorigenesis, the alternative splicing. 


\section{Acknowledgement}

This work was supported by Korea Research Foundation Grant (KRF-2001-015-FP0017).

\section{References}

Bottaro DP, Rubin JS, Faletto DL, Chan AM, Kmiecik TE, Vande Woude GF, Aaronson SA. Identification of the hepatocyte growth factor receptor as the c-met protooncogene product. Science 1991;251:802-4

Bowman T, Garcia R, Turkson J, Jove R. STATs in oncogenesis. Oncogene 2000;19:2474-88

Brathwaite O, Bayona W, Newcomb EW. p53 mutations in C57BL/6J murine thymic lymphomas induced by gammairradiation and N-methylnitrosourea. Cancer Res 1992;52: 3791-5

Bromberg JF, Wrzeszczynska MH, Devgan G, Zhao Y, Pestell RG, Albanese C, Darnell JE Jr. Stat3 as an oncogene. Cell 1999;98:295-303

Bussolino F, Di Renzo MF, Ziche M, Bocchietto E, Olivero M, Naldini L, Gaudino G, Tamagnone L, Coffer A, Comoglio PM. Hepatocyte growth factor is a potent angiogenic factor which stimulates endothelial cell motility and growth. J Cell Biol 1992;119:629-41

Calo V, Migliavacca M, Bazan V, Macaluso M, Buscemi M, Gebbia N, Russo A. STAT proteins: from normal control of cellular events to tumorigenesis. J Cell Physiol. 2003;197: $157-68$

Cao X, Tay A, Guy GR, Tan YH. Activation and association of Stat3 with Src in v-Src-transformed cell lines. Mol Cell Biol 1996;16:1595-603

Comoglio PM, Boccaccio C. The HGF receptor family: unconventional signal tansducers for invasive cell growth Genes. Cells 1996;1:347-54

Cooper CS, P.M., Blair DG, Tainsky MA, Huebner K, Croce CM, Vande Woude GF. Molecular cloning of a new transforming gene from a chemically transformed human cell line. Nature 1984;311:29-33

Gandino L, L. P., Medico E, Prat M, Comoglio PM. Phosphorylation of serine 985 negatively regulates the hepatocyte growth factor receptor kinase. J Biol Chem 1994;269:1815-20

Giles FJ, Vose JM, Do KA, Johnson MM, Manshouri T, Bociek G, Bierman PJ, O'Brien SM, Kantarjian HM, Armitage JO, Albitar M. Clinical relevance of circulating angiogenic factors in patients with non-Hodgkin's lymphoma or Hodgkin's lymphoma. Leuk Res 2004;28:595-604

Gohda E. Function and regulation of production of hepatocyte growth factor (HGF). Nippon Yakurigaku Zasshi 2002; 119:287-94, 309

Han SU, Lee JH, Kim WH, Cho YK, Kim MW. Significant correlation between serum level of hepatocyte growth factor and progression of gastric carcinoma. World J Surg 1999; 23:1176-80

Hsiao LT, Lin JT, Yu IT, Chiou TJ, Liu JH, Yen CC, Wang WS, Chen PM. High serum hepatocyte growth factor level in patients with non-Hodgkin's lymphoma. Eur J Haematol 2003;70:282-9

Jeffers M, Rong S, Vande Woude GF. Hepatocyte growth factor/scatter factor-Met signaling in tumorigenicity and invasion/metastasis. J Mol Med 1996a;74:505-13

Jeffers M, Rong S, Oskarsson M, Anver M, Vande Woude GF. Autocrine hepatocyte growth factor/scatter factor-Met signaling induces transformation and the invasive/metastastic phenotype in C127 cells. Oncogene 1996b;13:853-61

Jeffers M, Schmidt L, Nakaigawa N, Webb CP, Weirich G, Kishida T, Zbar B, Vande Woude GF. Activating mutations for the met tyrosine kinase receptor in human cancer. Proc Natl Acad Sci USA 1997;94:11445-50

Lee C, Yamada K. Identification of a novel type of alternative splicing of a tyrosine kinase receptor. Juxtamembrane deletion of the c-met protein kinase $\mathrm{C}$ serine phosphorylation regulatory site. J Biol Chem 1994;269: 19457-61

Lee C, Yamada K. Alternatively spliced juxtamembrane domain of a tyrosine kinase receptor is a multifunctional regulatory site. Deletion alters cellular tyrosine phosphorylation pattern and facilitates binding of phosphatidylinositol$3-\mathrm{OH}$ kinase to the hepatocyte growth factor receptor. J Biol Chem 1995;270:507-10

Lee JH, Han SU, Cho H, Jennings B, Gerrard B, Dean M, Schmidt L, Zbar B, Vande Woude GF A novel germ line juxtamembrane Met mutation in human gastric cancer. Oncogene 2000;19:4947-53

Lopez AJ. Alternative splicing of pre-mRNA: developmental consequences and mechanisms of regulation. Annu Rev Genet 1998;32:279-305

Montesano R., Schaller G, Orci L. Hepatocyte growth factor increases urokinase-type plasminogen activator (u-PA) and u-PA receptor expression in Madin-Darby canine kidney epithelial cells. Cell 1991;66:697-711

Nakamura T, T.H., Ichihara A. Purification and characterization of a growth factor from rat platelets for mature parenchymal hepatocytes in primary cultures. Proc Natl Acad Sci USA 1986;83:6489-93

Naldini L, Vigna E, Ferracini R, Longati P, Gandino L, Prat $M$, Comoglio PM. The tyrosine kinase encoded by the MET proto-oncogene is activated by autophosphorylation. Mol Cell Biol 1991;11:1793-803

Park M, D. M., Cooper CS, Schmidt M, O' Brien SJ, Blair DG, Vande Woude GF. Mechanism of met oncogene activation. Cell 1986;45:895-904

Park WS, D. S., Kim SY, Na EY, Shin MS, Pi JH, Kim BJ, Bae JH, Hong YK, Lee KS, Lee, SH, Y. N., Jang JJ, Pack $S$, Zhuang Z, Schmidt L, Zbar B, Lee JY. Somatic mutations in the kinase domain of the Met/hepatocyte growth factor receptor gene in childhood hepatocellular carcinomas. Cancer Res 1999;59:307-10

Schmidt L, Duh FM, Chen F, Kishida T, Glenn G, Choyke $P$, Scherer SW, Zhuang Z, Lubensky I, Dean M, Allilmets R, Chidambaram A, Bergerheim UR, Feltis JT, Casadevall C, Zamarron A, Bernues M, Richard S, Lips, C. J. M., Walter MM., Tsui LC, Geil L, Orcutt ML, Stackhouse T, Lipan J, Slife L, Brauch H, Decker J, Niehans G, Hughson MD, Moch 
H, Storkel S, Lerman MI, Linehan WM, Zbar B. Germline and somatic mutations in the tyrosine kinase domain of the MET proto-oncogene in papillary renal carcinomas. Nat Genet 1997;16:68-73

Skibinski G, Skibinska A, James K. Hepatocyte growth factor (HGF) protects c-met-expressing Burkitt's lymphoma cell lines from apoptotic death induced by DNA damaging agents. Eur J Cancer 2001;37:1562-9

Song L, Turkson J, Karras JG, Jove R, Haura EB. Activation of Stat3 by receptor tyrosine kinases and cytokines regulates survival in human non-small cell carcinoma cells. Oncogene 2003;22:4150-65

Stoker M, Gherardi E, Perryman M, Gray J. Scatter factor is a fibroblast-derived modulator of epithelial cell mobility. Nature 1987;327:239-42

Tanyi J, Tory K, Rigo J, Nagy B, Papp Z. Evaluation of the tyrosine kinase domain of the Met proto-oncogene in sporadic ovarian carcinomas. Pathol Oncol Res 1999;5: 187-91

Trovato M, Grosso M, Vitarelli E, Ruggeri RM, Alesci S, Trimarchi F, Barresi G, Benvenga S. Distinctive expression of STAT3 in papillary thyroid carcinomas and a subset of follicular adenomas. Histol Histopathol 2003;18:393-9

Tsarfaty I, Resau JH, Rulong S, Keydar I, Faletto DL, Vande Woude GF. The met proto-oncogene receptor and lumen formation. Science 1992;257:1258-61

Turkson J, Jove R. STAT proteins: novel molecular targets for cancer drug discovery. Oncogene 2000;19:6613-26

Vigna E, G. D., Longati P, Bardelli A, Comoglio PM. Loss of the exon encoding the juxtamembrane domain is essential for the oncogenic activation of TPR-MET. Oncogene 1999; $18: 4275-81$

Villa-Moruzzi EPF, Bardelli A, Vigna E, De Rosa S, Comoglio PM. Protein tyrosine phosphatase PTP-S binds to the juxtamembrane region of the hepatocyte growth factor receptor. Met Biochem J 1998;336:235-9

Yu CL, Meyer DJ, Campbell GS, Larner AC, Carter-Su C, Schwartz J, Jove R. Enhanced DNA-binding activity of a Stat3-related protein in cells transformed by the Src oncoprotein. Science 1995;269:81-3

Zhang YW, Wang LM, Jove R, Vande Woude GF. Requirement of Stat3 signaling for HGF/SF-Met mediated tumorigenesis. Oncogene 2002;21:217-26 